\title{
Propriétés ergodiques, en mesure infinie, de certains systèmes dynamiques fibrés
}

\author{
Y. GUIVARC'H \\ IRMAR, Mathematiques Université de Rennes I-35042 Rennes Cedex, France
}

(Received 20 July 1987 and revised 17 January 1989)

\begin{abstract}
We study the ergodic properties of a class of dynamical systems with infinite invariant measure. This class contains skew-products of Anosov systems with $\mathbb{R}^{d}$. The results are applied to the $K$ property of skew-products and also to the ergodicity of the geodesic flow on abelian coverings of compact manifolds with constant negative curvature.
\end{abstract}

\section{Introduction}

On considère ici des systèmes dynamiques ayant de fortes propriétés stochastiques comme c'est le cas des difféomorphismes d'Anosov ou du flot géodésique en courbure négative sur une variété compacte $V$. On étudie des systèmes dynamiques fibrés au-dessus de ces systèmes de base, la fibre étant le groupe $\mathbb{R}^{d}$ ou $\mathbb{Z}^{d}$; ces systèmes commutent donc avec l'action de $\mathbb{R}^{d}$ ou $\mathbb{Z}^{d}$, le quotient étant le système de base. Du point de vue mesurable, ces systèmes s'écrivent comme produits croisés à l'aide d'une fonction $f$ à valeurs dans $\mathbb{R}^{d}$ et les propriétés étudiées sont donc proches de celles des sommes de Birkhoff. Ce cadre permet d'englober diverses situations concrètes comme les marches aléatoires sur $\mathbb{R}^{d}$ avec mémoire [9] ou les flots géodésiques sur un revêtement abélien d'une variété compacte. Le modèle du gaz de Lorentz plan [5] appartient aussi à cette famille puisqu'il peut se présenter comme un flot géodésique (avec réflexion) sur un revêtement à fibre $\mathbb{Z}^{2}$ d'un tore plat bidimensionnel muni d'un obstacle circulaire réfléchissant. Les techniques développées ici ne permettent pas d'étudier cet exemple mais plutôt des situations beaucoup plus régulières à potential $\mathbb{Z}^{2}$-périodique et différentiable conduisant à des flots d'Anosov ou vérifiant l'axiome A de Smale [4].

Les propriétés étudiées correspondent dans le cas des marches aléatoires à des propriétés de théorie du potentiel comme la constance des harmoniques bornées [6] ou la finitude de la mesure potentiel et les preuves en sont inspirées; elles se relient ici à la propriété d'ergodicité en mesure infinie. Ces méthodes prolongent celles de [8] où sont étudiés les théorèmes limites du calcul des probabilités pour les systèmes envisagés ici.

Les résultats obtenus permettent de retrouver simplement des théorèmes établis en [18] concernant l'ergodicité de flots géodésiques, en restriction aux points non errants, sur des quotients non compacts de l'espace hyperbolique par des sous- 
groupes discrets. Ils permettent aussi d'établir la propriété $K$ pour des produits croisés en mesure finie, améliorant ainsi des résultats de [13]. Enfin, une application est donnée à l'étude des séries lacunaires du type $\sum_{0}^{\infty} z^{2^{k}}$.

Ces conclusions reposent sur l'étude préalable de la 'propriété $K$ ' ou de l'ergodicité pour des transformations fibrées au-dessus d'un sous-shift et la méthode du codage [2]. Par exemple le cas des systèmes d'Anosov s'en déduit rapidement.

La partie A décrit un certain nombre de notions préalables simples tandis que la partie $B$ est consacrée aux applications. Enfin, on justifie brièvement, en appendice le théorème limite local [8] qui intervient dans l'étude de l'ergodicité des systèmes cylindriques. Ce théorème limite local est aussi le point de départ des estimations de S. Kalikow [12] qui permettent de construire un exemple simple de transformation possédant la propriété $K$ mais non celle de Bernoulli. On discute la validité de telles estimations, dans un cadre plus général, qui pourrait permettre la construction d'exemples différentiables, construction qui a été effectuée par D. J. Rudolph [20] par une méthode n'utilisant pas toute la force des estimations de S. Kalikow.

Nous remercions vivement J.-P. Conze et J.-P. Thouvenot dont l'aide et les questions nous ont été très précieuses au cours de ce travail.

\section{Propriétés d'ergodicité}

1.1. Définitions. On considère un espace mesuré $E$, une mesure $\sigma$-finie $m$, une transformation $T$ préservant $m$. L'espace des fonctions $m$-intégrables sera noté $\mathbb{L}^{1}(m)$ et sera supposé séparable. Les mesures finies ayant une densité par rapport à $m$ seront identifiées aux éléments de $\mathbb{L}^{1}(\mathrm{~m})$ et, par exemple, l'ensemble des mesures de ce type ayant une masse nulle sera noté $\mathbb{L}_{0}^{1}(m)$; la variation totale d'une telle mesure $\alpha$ sera notée $\|\alpha\|_{1}$. La transformée de $\alpha$ par $T$ sera notée $\alpha T$. La mesure de Lebesgue sur $\mathbb{R}^{d}$ (ou $\mathbb{Z}^{d}$ ) sera notée $l$; une partition a de $E$ sera dite mesurable si la tribu naturelle sur l'espace quotient $E / a$ sépare les points de $E / a$. La tribu engendrée par $a$ sera encore notée $a$. L'image de $a$ par $T$, notée $T a$ est formée des images réciproques $T^{-1}(A)$ avec $A \in a$. On dira que $a$ est propre si le saturé, par rapport à $a$, de tout ensemble de mesure finie est encore de mesure finie. En ce cas on peut trouver des probabilités $m_{x}(x \in E)$ portées par les éléments de $a$ telle que $m$ se désintègre sous la forme

$$
m=\int_{E} m_{x} d m(x)=\int_{\bar{E}} \bar{m}_{\bar{x}} d \bar{m}(\bar{x})
$$

où $\bar{m}$ est la mesure image de $m$ sur $\bar{E}=E / a$. Si $T$ préserve $a$, c'est-à-dire $T a \subset a$ une transformation quotient $\bar{T}$ se trouve définie sur $\bar{E}$.

Enfin, si $T$ est un automorphisme, la partition a sera dite génératrice si les $T^{n} a$ ( $n \in \mathbb{Z}$ ) engendrent la tribu canonique de $E$. Ces définitions doivent être interprétées modulo la mesure $m$.

Définition 1. Soit $T$ un automorphisme de l'espace mesuré $(E, m)$, a une partition génératrice propre de $E$ qui est préservée par $T$. On dit que le système dynamique $(E, m, T)$ possède la propriété $K$ par rapport à $a$ si la tribu $\bigcap_{n \geq 0} T^{n} a$ est triviale. 
Définition 2. Soit $T$ un endomorphisme de $(E, m)$. On dit que le système $(E, m, T)$ est exact si pour tout $\alpha \in \mathbb{L}_{0}^{1}(m)$ on a $\lim _{n}\left\|\alpha T^{n}\right\|_{1}=0$.

$\mathrm{Si} \mathscr{B}$ est la tribu canonique sur $E$, l'exactitude de $(E, m, T)$ équivaut à la trivialité de la tribu asymptotique $\bigcap_{n \geq 0} T^{n} \mathscr{B}$. En effet, si la fonction bornée $F$ est asymptotique, elle s'écrit pour tout $n \geq 0: F=F_{n} \circ T^{n}$. Pour $\alpha \in \mathbb{L}_{0}^{1}(m)$ on $a$ :

$$
|\alpha(F)|=\left|\left(\alpha T^{n}\right)\left(F_{n}\right)\right| \leq\left\|\alpha T^{n}\right\|_{1}\left\|F_{n}\right\|_{\infty} \leq\left\|\alpha T^{n}\right\|_{1}\|F\|_{\infty}
$$

et l'hypothèse d'exactitude donne $\alpha(F)=0, F=$ cte.

Inversement, si $\lim _{n}\left\|\alpha T^{n}\right\|_{1}>0$ pour un certain $\alpha$ de $\mathbb{L}_{0}^{1}(m)$, on peut trouver des $F_{n}$ avec $\left\|F_{n}\right\|_{\infty}=1$ et $\left|\left(\alpha T^{n}\right)\left(F_{n}\right)\right| \geq \varepsilon>0$.

Soit $\left|\alpha\left(F_{n} \circ T^{n}\right)\right| \geq \varepsilon>0$. On peut trouver une sous-suite d'entiers $n$ telle que, pour tout $k F_{n} \circ T^{n-k}$ converge vers $F^{k}$ faiblement dans la dualité entre $\mathbb{L}^{1}$ et $\mathbb{L}^{\infty}$. Alors on a $F^{0}=F^{k} \circ T^{k}$ par passage à la limite faible à partir de $\left(F_{n} \circ T^{n-k}\right) \circ T^{k}=$ $F_{n} \circ T^{n}$. De plus $F^{0}$ est non constante car $\left|\alpha\left(F^{0}\right)\right|=\lim _{n}\left|\alpha\left(F_{n} \circ T^{n}\right)\right| \geq \varepsilon$. La tribu asymptotique est donc non triviale.

Ceci montre que si $T$ est un automorphisme de $(E, m)$ respectant la partition $a$, la propriété $K$ pour $(E, m, T)$ relativement à $a$ équivaut à l'exactitude du système quotient $(E / a, \bar{T}, \bar{m})$. Pour une situation voisine, on pourra consulter [16].

Exemple. Considérons une suite $X_{n}(n \in \mathbb{Z})$ de variables aléatoires indépendantes de même loi $p$ portées par une partie finie $S$ de $\mathbb{Z}$. Rappelons [23] que si les éléments de $S$ ne sont pas en progression arithmétique, les puissances de convolutions $p^{n}$ satisfont la propriété des suites moyennantes:

$$
\lim _{n} \sum_{k \in Z}\left|p^{n}(k+1)-p^{n}(k)\right|=0 .
$$

Ceci s'écrit encore $\lim _{n}\left\|p^{n} *\left(\delta_{1}-\delta_{0}\right)\right\|_{1}=0$ et on supposera ici cette propriété réalisée. Considérons le mesure produit $\pi=p^{\mathbf{Z}}$ sur $S^{\mathbf{Z}}$ et l'espace produit $E=S^{\mathbf{Z}} \times \mathbb{Z}$ muni de la mesure produit $\pi \times l$. Le décalage naturel sur $S^{Z}$ sera noté $\theta$, de sorte que $X_{k}=X_{0} \circ \theta^{k}$. A la marche aléatoire $s_{n}=\sum_{0}^{n-1} X_{k}$ de loi $p$ sur $\mathbb{Z}$ est associée la transformation $\tilde{\theta}$ sur $E$ définie par $\tilde{\theta}(\omega, t)=\left(\theta \omega, t+X_{0}(\omega)\right)$ et préservant la mesure $\pi \times l$. La décomposition d'une suite $\omega=\left(\omega_{k}\right)_{k \in \mathbf{Z}} \in S^{\mathbf{Z}}$ en $\omega^{-} \times \omega^{+}$avec $\omega^{-}=\left(\omega_{k}\right)_{k<0}$ et $\omega^{+}=\left(\omega_{k}\right)_{k \geq 0}$ fournit une décomposition $S^{\mathbf{Z}}=\Sigma^{-} \times \Sigma^{+}$et une partition $a$ en ensembles de la forme $\Sigma^{-} \times\left\{\omega^{+}\right\}$. Le système dynamique quotient de $(E, \tilde{\theta}, \pi \times l)$ sera défini par:

$$
\theta\left[\omega^{+}, t\right]=\left[\theta \omega^{+}, t+X_{0}\left(\omega^{+}\right)\right]
$$

et la mesure naturelle sur $\Sigma^{+} \times \mathbb{Z}$ sera encore notée $\pi \times l$. Montrons l'exactitude de ce dernier système, c'est-à-dire la propriété $K$ pour $(E, \theta, \pi \times l)$ par rapport à $a$. Soit $F$ une fonction asymptotique bornée: $F=F_{n} \circ \theta^{n}$ et posons $f_{n}(t)=$ $\int_{\Sigma^{+}} F_{n}(\omega, t) d \pi(\omega)$. Observons alors que

$$
\begin{aligned}
E\left[F / t, X_{0}\right. & \left.=x_{0}, X_{n}=x_{n}\right]=\int F\left(x_{0} \ldots x_{n} \omega, t\right) d \pi(\omega)=\int F_{n+1}\left[\omega, t+s_{n+1}(\omega)\right] d \pi(\omega) \\
& =f_{n+1}\left(t+s_{n+1}\right) .
\end{aligned}
$$

D'où en particulier $f_{0}(t)=\int f_{n}(t+y) d p^{n}(y)$. 
Notons aussi que, puisque $\tilde{\theta} E$ est de mesure pleine on a $F_{n}=F_{n+1} \circ \tilde{\theta}$ et donc comme plus haut:

$$
f_{n}(t)=\int f_{n+1}(t+y) d p(y)
$$

Ecrivons alors:

$$
\begin{gathered}
\left(\delta_{1}-\delta_{0}\right)\left(f_{0}\right)=\left(\delta_{1}-\delta_{0}\right)\left({ }^{v} p^{n} * f_{n}\right)=\left[p^{n} *\left(\delta_{1}-\delta_{0}\right)\right]\left(f_{n}\right), \\
\left|\left(\delta_{1}-\delta_{0}\right)\left(f_{0}\right)\right| \leqslant\left\|p^{n} *\left(\delta_{1}-\delta_{0}\right)\right\|_{1}\left\|f_{n}\right\|_{\infty} .
\end{gathered}
$$

Soit $f_{0}(0)=f_{0}(1)$ et plus généralement $f_{0}(k)=f_{0}(k+1)(k \in \mathbb{Z})$. Ceci donne $f_{0}=$ cte et plus généralement $f_{n}=$ cte $=f_{0}$. On a alors, puisque $F$ est bornée, par définition de $f_{n}$ :

$$
F(t, \omega)=\lim _{n} f_{n}\left(t+s_{n}\right)=\text { cte. }
$$

On va dans la partie 2 généraliser ce type de situation et étudier des analogues géométriques. La notion d'exactitude peut être relativisée comme suit:

Définition 3. Soit a une partition mesurable de $E$. On dira que $T$ est exacte relativement à $a$ si pour tout élément $\alpha$ de $\mathbb{L}^{1}(m)$, de projection nulle sur $E / a$ on a $\lim _{n}\left\|\alpha T^{n}\right\|_{1}=0$. On notera $\mathbb{L}_{a}^{1}(m)$ le noyau de la projection de $E$ sur $E / a$.

La proposition suivante découle alors des définitions:

Proposition 1. Soit a une partition mesurable de $(E, T, m)$ telle que $T_{a} \subset a$. On suppose $T$ exacte relativement à a et la transformation quotient $T$ sur $E /$ a exacte. Alors $T$ est exacte.

1.2. Consequences. On va considérer dans la suite des extensions d'un système dynamique $(X, \theta, \pi)$ par un groupe abélien que l'on prendra en général égal à $\mathbb{P}^{d}$.

Définition 4. Soit $(X, \theta, \pi)$ un système dynamique, $\pi$ étant une mesure $\theta$-invariante finie. Soit $f$ une fonction mesurable de $X$ dans $\mathbb{R}^{d}$. On appelle système cylindrique $(X, \theta, f)$ le système dynamique défini sur $X \times \mathbb{R}^{\mathrm{d}}$ par $\tilde{\theta}(x, t)=[\theta x, t+f(x)]$, la mesure invariante étant $\pi \times l$.

Pour un système cylindrique, la propriété, d'exactitude va se traduire sur $f$ ou plutot les sommes $S_{n}(x)=\sum_{0}^{n-1} f \circ \theta^{k}(x)$.

Définition 5. Soit $\mu_{n}$ une suite de probabilités sur $\mathbb{R}^{d}$. On dit que $\mu_{n}$ vérifie la propriété de moyenne si pour tout $\varphi \in \mathbb{L}_{0}^{1}(l)$ on a

$$
\lim _{n}\left\|\mu_{n} * \varphi\right\|_{1}=0 \text {. }
$$

Définition 6. Soit $(X, \theta, f)$ un système cylindrique. On dira que $f$ vérifie la propriété de moyenne si pout tout $y \in X$ et toute probabilité $\alpha \in \mathbb{L}^{1}(\pi)$ la loi ${ }^{n} \mu_{\alpha}^{y}$ de $S_{n}(x)=$ $\sum_{0}^{n-1} f \circ \theta^{k}(x)$ conditionnellement à $\theta^{n} x=y(x$ étant distribué suivant $\alpha)$ vérifie la propriété de moyenne. On peut alors énoncer la

Proposition 2. Soit $(X, \theta, f)$ un système cylindrique et supposons que $f$ vérifie la propriété de moyenne. Alors $\tilde{\theta}$ est exacte relativement à la partition en 'verticales' $\{x\} \times \mathbb{R}^{d}$. 
Preuve. Si $v$ est la partition en verticales il suffit de voir que pour un ensemble de mesures $\beta$ dense dans $\mathbb{L}_{v}^{1}(\pi \times l)$ on a

$$
\lim \left\|\beta \tilde{\theta}^{n}\right\|_{1}=0 \text {. }
$$

Soit $\xi \in \mathbb{L}_{0}^{1}(l)$ et $\alpha$ une probabilité à densité bornée de $\mathbb{L}^{1}(\pi)$. On a alors:

$$
(\alpha \times \xi) \tilde{\theta}^{n}=\int \delta_{y} \times\left({ }^{n} \mu_{\alpha}^{y} * \xi\right) d\left(\alpha \theta^{n}\right)(y)
$$

et

$$
\left\|(\alpha \times \xi) \tilde{\theta}^{n}\right\|_{1}=\int\left\|^{n} \mu_{\alpha}^{y} * \xi\right\|_{1} d\left(\alpha \theta^{n}\right)(y) .
$$

D'où par convergence dominée et en raison de la propriété de moyenne:

$$
\lim _{n}\left\|(\alpha \times \xi) \tilde{\theta}^{n}\right\|_{1}=0 \text {. }
$$

Justifions la propriété de totalité des éléments de la forme $\alpha \times \xi$ dans $\mathbb{L}_{v}^{1}(\pi \times \rho)$ : soit $g \in \mathbb{L}^{\infty}(\pi \times \rho)$ avec $\langle g, \alpha \times \xi\rangle=0$ ou

$$
\int g(x, t) \alpha(x) \xi(t) d \pi(x) d \rho(t)=0 .
$$

L'arbitraire de $\xi$ dans $\mathbb{L}_{0}^{1}(l)$ donne

$$
\int g(x, t) \alpha(x) d \pi(x)=\operatorname{cte}=c(\alpha)
$$

et enfin

$$
g(x, t)=c(x)
$$

avec

$$
c(\alpha)=\int \alpha(x) c(x) d \pi(x) .
$$

Le sous-espace des $\beta \in \mathbb{L}^{1}(\pi \times l)$ défini par $\langle g, \beta\rangle=0$ avec $g$ du type précédent n'est autre que $\mathbb{L}_{v}^{1}(\pi \times l)$, et ceci donne la conclusion voulue.

Proposition 3. Soit $(X, \theta, f)$ un système cylindrique exact. Soit $\left(Y, \mathbb{R}^{d}, \nu\right)$ un système dynamique ergodique, la mesure $\nu$ étant $\mathbb{R}^{d}$-invariante. Alors le produit croisé $X \times{ }_{f} Y$ est exact.

Preuve. Elle est analogue à celle de la Proposition 2.

Soit $\alpha \in \mathbb{L}^{1}(\pi), \xi \in \mathbb{L}_{0}^{1}(l), \rho \in \mathbb{L}^{1}(\nu)$. Notons le prolongement de l'action de $\mathbb{R}^{d}$ sur $Y$ à $\mathbb{L}^{1}(l)$ et $\mathbb{L}^{1}(\nu)$ par $*$.

Ainsi:

$$
\alpha \times(\rho * \xi) \tilde{\theta}^{n}=\int \delta_{y} \times\left(\rho *^{n} \mu_{\alpha}^{y} * \xi \mid d\left(\alpha \theta^{n}\right)(y)\right.
$$

et

$$
\begin{aligned}
\left\|\alpha \times(\rho * \xi) \tilde{\theta}^{n}\right\|_{1} & \leq \int\left\|^{n} \mu_{\alpha}^{y} * \xi\right\| d\left(\alpha \theta^{n}\right)(y)\|\rho\|_{1} \\
& =\left\|(\alpha \times \xi) \tilde{\theta}^{n}\right\|_{1}\|\rho\|_{1} .
\end{aligned}
$$


Comme $(X, \theta, f)$ est exact, on a bien $\lim _{n}\left\|\alpha \times(\rho * \xi) \tilde{\theta}^{n}\right\|_{1}=0$. L'ergodicité de l'action de $\mathbb{R}^{d}$ sur $Y$ implique, comme plus haut, la densité des éléments de la forme $\rho * \xi$ dans $\mathbb{L}_{0}^{1}(\nu)$ et on en conclut l'exactitude du produit croisé $X \times{ }_{f} Y$ relativement à la partition en verticales $\{x\} \times Y$. D'autre part l'exactitude de $(X, \theta, f)$ contient celle du facteur $(X, \theta, \pi)$ et la proposition 1 implique l'exactitude du produit croisé $\boldsymbol{X} \times \boldsymbol{f} Y$.

On a alors trivialement le

Corollatre. Supposons que le système $(X, \theta, \pi)$ possède la propriété $K$ relativement à la partition a, que $f$ soit une application a-mesurable de $X$ dans $\mathbb{R}^{d}$, que le système cylindrique $(X, \theta, f)$ possède la propriété $K$ par rapport à la partition en 'horizontales' $A \times\{t\}(A \in a, t \in \mathbb{R})$. Soit $\left(Y, \mathbb{R}^{d}, \nu\right)$ un système dynamique ergodique. Alors le produit croisé $X \times{ }_{f} Y$ possède la propriété $K$ par rapport à la partition en horizontales $A \times\{y\}$ $(A \in a, y \in Y)$.

Remarque. Dès que la propriété de moyenne est vraie pour $f$, définie sur $X / a$, la conclusion du corollaire est donc valable. Le résultat suivant correspond au théorème de Choquet-Deny [6].

Proposition 4. Supposons que $(E, T, m)$ vérifie la propriété $K$ par rapport à la partition a. Alors les fonctions bornées a-mesurable et T-invariantes sont constantes.

Preuve. $F$ étant invariante est également mesurable par rapport à la tribu $\bigcap_{n \geq 0} T_{a}^{n}$, donc constante d'après la propriété $K$.

1.3. Propriété de récurrence. On rappelle qu'un système dynamique $(E, T, m)$ est dit récurrent si pour tout ensemble non négligeable $A$, il existe $k>0$ avec $m\left(A \cap T^{-k} A\right)>0$. On montre aisément que la récurrence équivaut à l'absence d'ensembles errants, c-est-à-dire d'ensembles $D$ vérifiant $m(D)>0, T^{-k} D \cap D=\varnothing$ $\forall k>0$. Elle équivaut aussi à la propriété: si le borélien $B$ satisfait $T^{-1} B \subset B$, on a $T^{-1} B=B$. Enfin rappelons que si $T$ est inversible et $m$ non atomique, l'ergodicité implique la récurrence.

Proposition 5. Supposons que le système dynamique $(E, T, m)$ possède la propriété $K$ par rapport à la partition a et que la système quotient $(E / a, \bar{T}, \bar{m})$ soit récurrent. Alors le système $(E, T, m)$ est ergodique.

Lemme. Soit $(X, S, \rho)$ un système dynamique où $\rho$ est finie, a une sous-tribu des boréliens de $X$ par rapport à laquelle $S$ est mesurable, $f$ un élément $S$-invariant de $\mathbb{L}^{2}(X, \rho)$. Alors l'espérance conditionnelle $f=E[f / a]$ est $S$-invariante.

Preuve. Soit $\hat{a}$ le sous-espace fermé de $\mathbb{L}^{2}(X, \rho)$ engendré par $a$. Puisque $S$ est une isométrie, la projection $S \tilde{f}$ de $S f$ sur $S a$ est l'image par $S$ de la projection $\bar{f}$ de $f$ sur $\hat{a}: \tilde{f}=S \tilde{f}=S \tilde{f}$. Par ailleurs, puisque $S \hat{a} \subset \hat{a}, \tilde{f}$ est la projection de $\bar{f}$ sur $S \hat{a}$. Comme la norme de $\tilde{f}$ est égale à celle de $S \bar{f}$, donc à celle de $\bar{f}$, on en conclut: $\bar{f}=\tilde{f}=S \tilde{f}$.

Preuve de la proposition. On se ramène à des transformations induites sur des ensembles de mesure finie. Soit $\bar{A}$ un borélien de mesure finie dans $E / a$; la propriété de récurrence permet de définir la transformation induite $\bar{T}_{\bar{A}}$ et on va montrer son 
ergodicité. Si $\bar{C} \subset \bar{A}$ est $\bar{T}_{\bar{A}}$-invariant, on a en posant

$$
\bar{B}=\bigcup_{0}^{\infty} \bar{T}^{-k} \bar{C}: \quad \bar{T}^{-1} \bar{B} \subset \bar{B}, \quad \bar{B} \cap \bar{A}=\bar{C} .
$$

La propriété de récurrence donne $\bar{T}^{-1} \bar{B}=\bar{B}$ et la Proposition 4 donne que si $\bar{m}(\bar{C})>0$, on $a: \bar{B}=E / a, \bar{A}=\bar{C}$. Soit maintenant $A$ l'image réciproque de $\bar{A}$ dans $E$. Si $\tau_{A}(x)$ est le temps de retour de $x \in A$ dans $A$, il est clair que $\tau_{A}$ est $a$-mesurable puisque $T$ et $A$ le sont; la transformation induite $T_{A}$ par $T$ sur $A$ est bien définie et correspond à $\bar{T}_{\bar{A}}$. Montrons l'ergodicité de $T_{A}$; soit $C \subset A$ un ensemble $T_{A^{-}}$ invariant et $\bar{f}=E\left[1_{C} / a\right]$; le lemme montre que $\bar{f}$ est $\bar{T}_{\bar{A}}$-invariante donc constante et égale à $m(C)$. Le même argument vaut pour $\bar{f}_{n}=E\left[1_{C} / T^{-n} a\right](n \geq 0)$ et donne $\bar{f}_{n}=m(C)$ et comme $a$ est génératrice on en conclut $1_{C}=\lim \bar{f}_{n}=m(C)$, soit $C=A$ ou $\varnothing$. D'autre part l'ensemble $B=\bigcup_{0}^{\infty} T^{-k} A$ appartient à la tribu $a$ et vérifie $T^{-1} B \subset B$; la propriété de récurrence dans $E / a$ jointe à la propriété $K$ donne alors $E=\bigcup_{0}^{\infty} T^{-k} A$. Notons que ces propriétés d'être ergodique et de générer $E$ valent pour $T^{-p} A$ aussi bien que $A(p \geq 0)$. Si maintenant $D \subset E$ est un ensemble $T$. invariant négligeable on a $m\left(D \cap T^{-p} A\right)>0$ pour un $p$ au moins, par exemple $m(D \cap A)>0$. L'ensemble $D \cap A$ est $T_{A}$-invariant et par ergodicité de $T_{A}$ on en conclut $D \cap A=A$ et $D=\bigcup_{0}^{\infty} T^{-k} D=\bigcup_{0}^{\infty} T^{-k} A=E$.

Il sera commode dans la suite d'étudier la récurrence d'un système, en général non inversible, à l'aide de l'opérateur adjoint $T^{*}$ de $T$ qui est une contraction de $\mathbb{L}^{\infty}(m)$. On considère alors le noyau potentiel de $T^{*}: G(x, B)=\sum_{0}^{\infty}\left(T^{*}\right)^{k} 1_{B}(x)=$ $G 1_{B}(x)$.

\section{Proposition 6}

(a) S'il existe une partie $B$ de $E$ de mesure finie telle que $G(x, B)=+\infty p p$, alors $(E, T, m)$ est récurrent.

(b) Si pour une partie B non négligeable de $E, G(x, B)$ est bornée, le système $(E, T, m)$ n'est pas récurrent.

Preuve.

(a) Soit $D$ un ensemble errant, donc tel que $T^{-k} D \cap D=\varnothing \quad \forall k>0$. Alors $\sum_{0}^{\infty} 1_{D} \circ T^{k} \leq 1$ et: $m(B) \geq \sum_{0}^{\infty}\left\langle 1_{D} \circ T^{k}, 1_{B}\right\rangle=\left\langle 1_{D}, G 1_{B}\right\rangle$. Donc $G(x, B)$ est finie sur $D$, ce qui montre d'après l'hypothèse que $m(D)=0$.

(b) Posons $h=\sum_{0}^{\infty} 1_{B} \circ T^{k}$ et observons que $\left\{h, 1_{B}\right\rangle=\left\langle 1_{B}, G 1_{B}\right\rangle<+\infty$. On a d'autre part $h \circ T+1_{B}=h$ et $h \circ T \leq h$. On en déduit que l'ensemble $C=\{h \geq c\}$ vérifie $T^{-1} C \subset C$ avec inégalité stricte pour un certain $c>0$. On ne peut donc avoir la récurrence.

\section{Systèmes cylindriques et applications}

2.1. Propriétés de périodicité. Soit $(X, \theta, \pi)$ un système dynamique, avec $\pi$ mesure $\theta$-invariante finie, $\mathbb{L}^{1}\left(\pi, \mathbb{R}^{d}\right)$ l'espace des fonction intégrables à valeurs dans $\mathbb{R}^{d}$.

Définition 1. On dira que $f$ et $g$ fonctions de $L^{1}\left(\pi, \mathbb{R}^{d}\right)$ sont équivalentes s'il existe $\varphi \in \mathbb{L}^{1}\left(\pi, \mathbb{R}^{d}\right)$ avec $f-g=\varphi \circ \theta-\varphi$.

Si $f$ est équivalente à o elle sera dite triviale, et ceci correspond au fair que les deux systèmes cylindriques $(X, \theta, f)$ et $(X, \theta, g)$ s'identifient $\operatorname{par}(x, t) \rightarrow[x, t+\varphi(x)]$. 
Des notions de périodicité analogues à celles intervenant pour les marches aléatoires s'introduisent ici.

Définition 2. On dira que $f \in \mathbb{L}^{1}\left(\pi, \mathbb{R}^{d}\right)$ est arithmétique si elle est équivalente à $g \in \mathbb{L}^{1}\left(\pi, \mathbb{R}^{d}\right)$ telle que $g(X)$ soit contenu dans un sous-groupe fermé strict de $\mathbb{R}^{d}$.

Définition 3. On dira que $f \in \mathbb{L}^{1}\left(\pi, \mathbb{R}^{d}\right)$ est strictement apériodique s'il n'existe pas de constante $c$ telle que $f-c$ soit arithmétique.

Remarques. (1) Les systèmes cylindriques que l'on va considérer seront des deux types suivants: (a) Le couple $(X, \theta)$ sera un sous-shift de type fini noté $(\Sigma, \theta)$, supposé topologiquement transitif [2], $f$ sera une fonction Holdérienne et le mesure $\pi$ sera un état d'équilibre [2] associé à une fonction holdérienne que l'on appellera ici mesure de Gibbs. On utilisera essentiellement les résultats de [2] et [4]. Dans ce cas l'équivalence entre $f$ et $g$ holdérienne permet de trouver $\varphi$ holdérienne $([2,8])$ avec $f-g=\varphi \circ \theta-\varphi$. (b) Le couple $(X, \theta)$ sera un ensemble basique hyperbolique $(\Omega, u)$ [2] pour un difféomorphisme u d'une variété $V, f$ sera holdérienne comme en a) et $\pi$ de Gibbs également. Le même conclusion qu'en (a) vaut pour l'équivalence: $\varphi$ sera holdérienne $\operatorname{sur} \Omega([2,15])$.

(2) Les conditions d'arithméticité et de stricte apériodicité se formuleront mieux en termes de caractères de $\mathbb{R}^{d}$, pour une fonction $f$ holdérienne sur $\Sigma$ ou $\Omega$. (a) $f$ sera arithmétique [resp. non strictement apériodique] s’il existe un caractère non trivial de $\mathbb{R}^{d}$ ou $\mathbb{Z}^{d}: e_{\lambda}(x)=\mathrm{e}^{i<\lambda, x)}\left(\lambda \in \mathbb{R}^{d}\right)$ et une fonction $\varphi$ holdérienne de $\Sigma$ dans $\mathrm{T}[8]$ avec $\mathrm{e}^{i\langle\lambda, f\rangle}=(\varphi \circ \theta) / \varphi$ [resp. $e^{i\langle\lambda, f\rangle}=e^{i \alpha}[(\varphi \circ \theta) / \varphi$ ] avec $\alpha \in \mathbb{R}]$. (b) Le remplacement de $\Sigma$ par l'ensemble basique hyperbolique $\Omega$ n'affecte pas le résultat précédent: $\varphi$ est holdérienne sur $\Omega$. Il suffit clairement d'examiner le premier cas et l'on code $(\Omega, u)$ par un sous-shift de type fini $(\Sigma, \theta)$, les images réciproques par le codage $\eta$ d'un point de $\Omega$ étant en nombre borné par $N<+\infty$ : d'après (a) on obtient une fonction $\Psi$ holdérienne sur $\Sigma$ et $\lambda \in \mathbb{R}^{d}$ avec

$$
e^{i\left(\lambda, f^{\prime}\right\rangle}=(\Psi \circ \theta) / \Psi \text { où } f^{\prime}=f_{0}
$$

si alors $x$ est un point périodique de période $n$ de $\Omega$, tout point $\omega \in \Sigma$ avec $\eta(\omega)=x$ sera périodique de periode $N n$ au plus. On en déduit:

$$
\prod_{k=0}^{N_{n-1}} e^{i\left\langle\lambda, f^{\prime \prime} \circ \theta^{k}(\omega)\right\rangle}=\prod_{k=0}^{n-1} e^{i N\left\langle\lambda, f \circ u^{k}(x)\right\rangle}=1
$$

On peut alors montrer que

$$
e^{i\langle\lambda, N f\rangle}=(\varphi \circ u) / \varphi
$$

avec $\varphi$ holdérienne sur $\Omega$. Il suffit pour cela d'étendre le théorème 3 de [15] ou de répéter en termes multiplicatifs l'argument de [2] pp. 93-94 qui permet de construire une fonction holdérienne $\varphi$ vérifiant $f=\varphi \circ u-\varphi$ dès que les sommes $\sum_{0}^{n-1} f \circ u^{k}(x)$ $s$ 'annulent sur les trajectoires périodiques de période $n$, la fonction $f$ étant remplacée $\operatorname{par} e^{i\langle\lambda, N f\rangle}$.

(3) Dans le cas d'un sous-shift on peut remplacer $f$ par une fonction holdérienne équivalente $f^{\prime}$ ne dépendent que des coordonnées positives et donc définie sur $\Sigma^{+}$. Le système cylindrique $\left(\Sigma, \theta, f^{\prime}\right)$ possède donc une partition mesurable $\theta$-invariante 
qui est formée des ensembles du type $\Sigma^{-} \times\left\{\omega^{+} \times t\right\}$ où $\omega^{+} \in \Sigma^{+}, t \in \mathbb{R}$. Par l'isomorphisme $(\omega, t) \rightarrow[\omega, t+\varphi \circ \theta(\omega)-\varphi(\omega)]$, le système $\left(\Sigma, \theta, f^{\prime}\right)$ s'identifie à $(\Sigma, \theta, f)$ et la partition invariante précédente se transforme en une nouvelle partition que l'on dira 'partition naturelle'.

Définition. Soit $(E, d)$ un espace métrique muni d'une transformation continue $T$. On appellera feuille stable de $x \in E$ l'ensemble $V(x)=\left\{y: \lim d\left(T^{n} y, T^{n} x\right)=0\right\}$.

Conséquences. L'ensemble $E$ est donc réunion disjointe de ses feuilles stables; dans le cas des systèmes cylindriques examinés plut haut, les feuilles stables se décrivent aisément et des partitions naturelles leur sont associées.

Pour le système $\left(\Sigma, \theta, f^{\prime}\right)$ la feuille stable de $(\omega, t) \in \Sigma \times \mathbb{R}^{d}$ n'est autre que l'ensemble des $(\sigma, t)$ avec $\sigma \in \Sigma$ ayant le même bout gauche que $\omega$; chaque feuille stable est réunion disjointe d'éléments de la partition naturelle. Les mémes propriétés valent pour $(\Sigma, \theta, f)$ : les feuilles stables de $\Sigma$ se relèvent naturellement dans $\Sigma \times \mathbb{R}^{d}$ en feuilles stables. Pour le système $(\Omega, u, f)$ associé à un ensemble basique hyperbolique le codage $\eta: \Sigma \rightarrow \Omega$ transforme les feuilles stables de $\Sigma$ en variétés stables de $\Omega$. Les feuilles stables du système cylindrique $(\Sigma, \theta, f \circ \eta)$ ont alors pour images dans $(\Omega, u, f)$ les feuilles stables de ce dernier système; les variétés stables de $(\Omega, u)$ se relèvent donc naturellement dans $\Omega \times \mathbb{R}^{d}$ en variétés stables. On peut considérer sur $\Omega \times \mathbb{R}^{d}$ la mesure produit de la mesure Gibbs $\pi$ sur $\Omega$ par la mesure de Lebesgue $l$ sur $\mathbb{R}^{d}$; la partition naturelle de $\sum \times \mathbb{R}^{d}$ se transporte alors en une partition invariante que l'on dira également canonique. Chaque variété stable est réunion disjointe (modulo $\pi \times l$ ) d'éléments de la partition canonique.

2.2. Propriétés de moyenne. On est donc ramené par ce qui précède, en ce qui concerne la propriétè $K$ par exemple, à l'étude d'un système cylindrique $\left(\Sigma^{+}, \theta, f\right)$ où $f$ est holdérienne sur $\Sigma^{+}$et strictement apériodique. Afin d'établir la propriété de moyenne pour $f$, justifions deux lemmes.

Lemme 1. Soit $\mu_{n}$ une suite de probabilités sur $\mathbb{R}^{d}$, portées par des compacts $K_{n}$. On suppose que $\lim _{n} \rho\left(K_{n}\right)^{1 / n}=1$ et que, pour tout caractère $\lambda \neq 0$ de $\mathbb{R}^{d}$ on $a$ $\overline{\lim }_{n}\left|\hat{\mu}_{n}(\lambda)\right|<1$. Alors $\mu_{n}$ possède la propriété de moyenne.

Preuve. On sait que les éléments $\varphi \in \mathbb{L}_{0}^{1}(l)$ dont la transformée de Fourier est à support compact sont denses dans $\mathbb{L}_{0}^{1}(l)$. Il suffit donc de voir que, pour de tels $\varphi$ on $a$ :

$$
\lim _{n}\left\|\mu_{n} * \varphi\right\|=0
$$

Examinons d'abord $\left\|\mu_{n} * \varphi\right\|_{2}$ puisque $\varphi \in \mathbb{L}^{2}(l)$ :

$$
\left\|\mu_{n} * \varphi\right\|_{2}^{2}=\int\left|\hat{\mu}_{n}(\lambda)\right|^{2}|\varphi(\lambda)|^{2} d \lambda
$$

comme $\varphi(\lambda)$ est bornée et à support compact on a

$$
\left\|\mu_{n} * \varphi\right\|_{2} \leq C \rho^{n}
$$

pour un certain $C$ et un $\rho$ tel que $\varlimsup_{n}\left|\hat{\mu}_{n}(\lambda)\right|^{1 / n}<\rho<1$. Par ailleurs l'inégalité de 
Schwarz donne:

$$
\left\|\mu_{n} * \varphi\right\|_{1} \leq l\left(K_{n}\right)^{1 / 2}\left\|\mu_{n} * \varphi\right\|_{2}
$$

d'où la conclusion puisque $l\left(K_{n}\right)$ est à croissance non exponentielle.

Considérons l'action de $\tilde{\theta}$ sur les fonctions de la forme $\varphi(x) e_{\lambda}(x)$ avec $\varphi \in \mathbb{L}^{\prime}\left(\pi^{+}\right)$ et $e_{\lambda}(x)=e^{i\langle\lambda, x\rangle}$ caractère de $\mathbb{R}^{d}$. La formule $\tilde{\theta}\left(\varphi \cdot e_{\lambda}\right)=\left(\theta_{\lambda} \varphi\right) \cdot e_{\lambda}$ où $\theta_{\lambda} \varphi=$ $e^{i(\lambda, f)}(\varphi \circ \theta)$ définit une contraction de $\mathbb{L}^{1}\left(\pi^{+}\right)$dont on notera l'adjoint sur $L^{\infty}\left(\pi^{+}\right)$ par $P_{\lambda}$. En particulier $P_{0}=P$ est l'adjoint de $\theta$ et l'on a:

$$
P_{\lambda} \varphi=P\left[e^{i(\lambda, f)} \varphi\right] \text {. }
$$

On a vu en [8] que la restriction de $P_{\lambda}$ à l'espace de Banach $H_{\alpha}$ des fonctions holdériennes d'ordre $\alpha$ est bien définie et quasi-compacte. De plus la condition de stricte apériodicité de $f$ équivaut à l'absence de valeurs propres de module 1 pour $P_{\lambda}(\lambda \neq 0)$.

On a donc $\lim _{n}\left\|P_{\lambda}\right\|^{1 / n}<1(\lambda \neq 0)$.

LeMME 2. Soit $\alpha$ un élément de $\mathbb{L}^{1}\left(\pi^{+}\right)$et considérons la désintégration verticale de la mesure $\left(\alpha \pi^{+}\right) \tilde{\theta}^{n}:\left(\alpha \cdot \pi^{+}\right) \tilde{\theta}^{n}=\int \delta_{y} \times{ }^{\alpha} \mu_{n}^{y} d \pi^{+}(y)$.

Alors

$$
\widehat{{ }^{\alpha} \mu_{n}^{y}}(\lambda)=P_{\lambda} \alpha(y) .
$$

Preuve. Il suffit de voir $\widehat{{ }^{\alpha} \mu_{1}^{y}}(\lambda)=P_{\lambda} \alpha(y)$.

Or:

$$
\left[\left(\alpha \cdot \pi^{+}\right) \tilde{\theta}\right]\left(\varphi \cdot e_{\lambda}\right)=\left[\alpha \cdot \pi^{+}\right]\left(\theta_{\lambda} \varphi \cdot e_{\lambda}\right)=\left[\alpha \cdot \pi^{+}\right]\left(\theta_{\lambda} \varphi\right)=\int P_{\lambda} \alpha(y) \varphi(y) d \pi^{+}(y)
$$

Aussi:

$$
\left[\left(\alpha \cdot \pi^{+}\right) \tilde{\theta}\right]\left(\varphi \cdot e_{\lambda}\right)=\int\left(\delta_{y} \times^{\alpha} \mu_{1}^{y}\right)\left(\varphi \cdot e_{\lambda}\right) d \pi^{+}(y)=\int \varphi(y)^{\alpha} \mu_{1}^{y}(\lambda) d \pi^{+}(y) .
$$

D'où:

$$
\widehat{\mu_{\mu}^{y}}(\lambda)=P_{\lambda} \alpha(y) \text {. }
$$

On a alors le:

THÉORÈME. Soit $f$ une fonction holdérienne à valeurs dans $\mathbb{R}^{d}$ définie sur le sous-shift $\Sigma^{+}$. On suppose $f$ strictement apériodique. Alors $f$ possède la propriété de moyenne.

Preuve. Il suffit d'observer que ${ }^{\alpha} \mu_{n}^{y}$ possède la propriété de moyenne car les hypothèses du Lemme 1 sont satisfaites: d'après la stricte apériodicité de $f$

$$
\varlimsup_{n}\left|\widehat{\alpha} \mu_{n}^{y}(\lambda)\right| \leq \varlimsup_{n}\left\|P_{\lambda} \alpha\right\|^{1 / n}<1
$$

et d'autre part le support de ${ }^{\alpha} \mu_{n}^{y}$ a au plus pour diamètre $2 n\|f\|_{\infty}$.

2.3. Sous-shifts et systèmes d'Anosov. Considérons d'abord un sous-shift $\Sigma$ de type fini muni d'une mesure de Gibbs $\pi$ et reprenons les notations déjà introduites. Le 
théorème suivant et ses deux premiers corollaires découlent immédiatement du paragraphe précédent et de la partie $\mathrm{A}$.

THÉORÈME. Soit $f$ une fonction holdérienne à valeurs dans $\mathbb{R}^{d}$, définie sur le sous-shift $\Sigma$ et supposons $f$ strictement apériodique. Alors le système cylindrique $(\Sigma, \theta, f)$ possède la propriété $K$ par rapport à la partition naturelle.

Corollaire 1. Avec les notations du théorème, considérons de plus une fonction bornée $F$ sur $\Sigma \times \mathbb{R}^{d}$ qui est continue en restriction aux éléments de la partition naturelle. Alors $F=$ cte.

COROLlaire 2. Soit $\left(Y, \mathbb{R}^{d}, \nu\right)$ un système dynamique où $\nu$ est une mesure finie $\mathbf{R}^{d}$-invariante, $f$ une fonction holdérienne sur $\Sigma$, à valeurs dans $\mathbb{R}^{d}$, qui est supposée strictement apériodique. Alors le produit croisé $\Sigma \times{ }_{f} Y$ possède la propriété $\mathrm{K}$.

Remarque. La partition sur $\Sigma \times{ }_{f} Y$ s'obtient à l'aide de la partition sur $\Sigma$, par l'application $(\omega, t) \rightarrow(\omega, t \cdot y)$

Corollaire 3. Avec les notations du théorème, supposons de plus $f$ d'intégrale nulle. Alors le système cylindrique $(\Sigma, \theta, f)$ est ergodique si et seulement si $d=1$ ou 2.

Preuve. On peut supposer $f$ définie sur $\Sigma^{+}$, et il suffit d'établire la récurrence ou la non-récurrence suivant $d \leq 2$ ou $d>2$, ceci d'après la Proposition 5 de 1 . Si $\tilde{P}$ désigne le noyau adjoint de $\tilde{\theta}$, le théorème local de [8], appliqué à la chaîne de noyau $\tilde{P}$ et à la fonction $f$ donne [cf Appendice]:

$$
\tilde{P}^{n}\left[(x, t), \Sigma^{+} \times B\right] \sim \mathrm{cte} / n^{d / 2}
$$

pour tous $(x, t)$ et toute boule $B$. On en conclut $G\left[(x, t), \Sigma^{+} \times B\right]=\infty$ si $d \leq 2$ et $G\left[(x, t), \Sigma^{+} \times B\right]$ bornée sinon. Il suffit donc d'appliquer la Proposition 6 de $\mathrm{A}$.

On peut maintenant grâce aux remarques de la partie 1 donner des résultats analogues aux précédents en remplaçant $\Sigma$ par un ensemble basique hyperbolique $\Omega$ en vertu des isomorphismes mesurables notés plus haut.

THÉORÈME. Soit $V$ une variété compacte, $u$ un difféomorphisme de $V, f$ une fonction holdérienne sur $V$ à valeurs dans $\mathbb{R}^{d}, \Omega$ un ensemble basique hyperbolique pour $u$ et l'on considère le système cylindrique $(\Omega, u, f)$ muni de la mesure $\pi \times l$ où $\pi$ est une mesure de Gibbs, $f$ strictement apériodique, c'est-à-dire qu'il n'existe pas $\varphi$ holdérienne de $\Omega$ dans $T, \lambda \in \mathbb{R}^{d}$ et $\alpha \in \mathbb{R}$ avec (sur $\Omega$ )

$$
e^{i(\lambda, f)}=e^{i \alpha}[(\varphi \circ u) / \varphi]
$$

Alors le système cylindrique $(\Omega, u, f)$ possède la propriété $K$ vis-à-vis de la partition naturelle.

Corollaire 1. Avec les notations du théorème, considérons de plus une fonction bornée $F$ sur $\Omega \times \mathbb{R}^{d}$ qui est continue en restriction aux éléments de la partition naturelle. Alors $F=$ cte. 
Remarque. En particulier si $F$ est continue en restriction aux variétés stables de $\Omega \times \mathbb{R}^{d}$, on a $F=$ cte.

Corollaire 2. Avec les notations du théorème, soit $\left(Y, \mathbb{R}^{d}, \nu\right)$ un système dynamique ergodique où $\nu$ est une mesure finie $\mathbb{R}^{d}$-invariante, $f$ une fonction holdérienne sur $V$, ̀̀ valeurs dans $\mathbb{R}^{d}$ qui est supposée strictement apériodique. Alors le produit croisé $V \times{ }_{f} Y$ muni de la mesure $\pi \times \nu$, possède la propriété $K$.

Remarques. Un théorème de ce type a été obtenue par A. Katok [13] en utilisant les méthodes des systèmes partiellement hyperboliques, dans le cas d'un système d'Anosov de base.

Des systèmes de ce type ont été l'objet d'études approfondies en théorie ergodique, en particulier dans le cadre des ' $\left(T, T^{-1}\right)$-transformations'. Une telle transformation est définie sur $\Sigma \times Y$, où $(Y, T, \nu)$ est un système dynamique ergodique $\Sigma=\{-1,1\}^{Z}$ est muni de la mesure produit de paramètre $\frac{1}{2}$ par:

$$
T(\omega, y)=\left[\theta \omega, T^{\omega_{0}} y\right] \text {. }
$$

Le cas où $(Y, T, \nu)$ est une rotation est étudié en [1] et [17], celui où $(Y, T, \nu)$ est lui-même un shift de Bernoulli en [12]. Cette dernière situation fournit des exemples de systèmes possédant le propriété $K$ mais non celle de Bernouilli et la preuve est basée sur les estimations du théorème local. Un tel théorème, dans le cadre général étudié ici, a été obtenu en [8] [cf Appendice].

Corollaire 3. Avec les notations du théorème supposons de plus $f d$ 'intégrale nulle. Alors le système cylindrique $(V, u, f)$ est $\pi \times$ l ergodique si et seulement si $d=1$ ou 2 .

\section{Remarques}

(1) On voit aisément que la condition de stricte apériodicité dans les Corollaires 1 et 3 des deux théorèmes présédents peut être remplacée par celle de non arithméticité, c'est à dire la non existence de $\lambda \in \mathbb{R}^{d}$ et d'une fonction holdérienne $\varphi$ à valeurs dans $\boldsymbol{T}$ avec

$$
e^{i\langle\lambda, f\rangle}=(\varphi \circ \theta) / \varphi
$$

(2) Des questions voisines de celles traitées ici ont été étudiées en [8] et [9]. L'exemple de la transformation dilatante $z \rightarrow z^{2}$ de $\mathbb{T}$ dans $T$ et de la fonction $f(z)=z$ a été mentionné du point de vue de la récurrence. On obtient donc ici l'ergodicité du système cylindrique correspondant $(z, t) \rightarrow\left(z^{2}, t+z\right)$ qui génère les sommes $\sum_{0}^{n-1} z^{2^{k}}$ dans $\mathbb{C}$.

2.4. Flots D'Anosov et flots Géodesiques. On retrouve ici comme conséquences des résultats précédents des théorèmes d'ergodicité pour certains flots géodésiques en mesure infinie [18]. En fait ces résultats sont des cas particuliers d'un théorème concernant les flots d'Anosov ou plus généralement les flots vérifiant l'axiome $\mathrm{A}$ de Smale [4].

(1) Nombre de tours d'un flot. On considère ici une variété riemannienne $V$, un revêtement abélien $W$ à fibre $\Gamma$ isomorphe à $\mathbb{Z}^{d}$, un flot différentiable $g^{t}$ sur $V$ et on note $p$ la projection de $W$ sur $V$. On considère aussi un compact invariant $\Lambda$ de $V$ qui est basique hyperbolique pour le flot $g^{\prime}$. Le flot $g^{\prime}$ se relève sur $W$ et sera 
encore noté $g^{t}$. On va définir le nombre de tours du flot $g^{t}$ relativement à une mesure de Gibbs (portée par $\Lambda$ ) et au revêtement $W$. On se fixe un isomorphisme du groupe $\Gamma$ dans un espace vectoriel $\mathbb{R}^{d}$ tel que l'image de $\Gamma$ soit un réseau de $\mathbb{R}^{d}$. Il est connu qu'un choix canonique pour cet espace vectoriel est un certain quotient du groupe d'homologie à coefficients réels de $V$ mais ces notions n'interviendront pas ici, grâce à la structure des flots hyperboliques [4]. On se donne aussi un point $a \in V$ et une famille de chemins continus joignant a aux points du support de $\mu$; on peut alors définir pour tout $v \in \Lambda$ et $A \in \mathbb{R}$ un chemin fermé en joignant a aux extrémités du chemin $t \rightarrow g^{\prime} v(0 \leq t \leq A)(v \in \Lambda)$. Ce chemin se relève dans $W$ et les extrémités se déduisant par l'isométrie $\gamma_{A} \in \Gamma$. Le nombre de tours envisagé $e_{\mu}^{w}$ se définit alors comme la limite $(p * p)$ de $(1 / A) \gamma_{A}$ lorsque $A$ tend vers l'infini, limite dont on va ici justifier l'existence par une analyse du flot $g^{t}$. Il est clair que cette limite ne dépend pas de a ni des chemins joignant a au support de $\mu$. Rappelons [4] que l'ensemble basique $\Lambda$ admet un codage par le flot spécial $C=(\Sigma, h)$ où $\Sigma$ est un sous-shift de type fini topologiquement mélangeant et $h$ une fonction holdérienne sur $\Sigma$, que le codage $\eta: C \rightarrow \Lambda$ est également holdérien et que les images réciproques des points de $\Lambda$ ont un cardinal borné par $N$. Construisons alors le fibré image réciproque de $W$ qui est encore un fibré holdérien $D$ au-dessus de $C$, les fibres s'identifiant à $\Gamma$ :

$$
D=\{(c, w) \in C \times W ; \eta(c)=p(w)\} .
$$

Le flot $g^{t}$ agit encore sur $D$ en commutant avec $\Gamma$ et sera noté $g^{t}$; on notera $q$ la projection de $D$ sur $C$. Soit $\Delta$ le sous-fibré de $D$ correspondant à $\Sigma$, c'est-à-dire $\Delta=q^{-1}(\Sigma)$ et posons $k(\delta)=h(\sigma)$ pour $\delta=(\sigma, w) \in \Delta \subset D$. Il est clair que $D$ s'identifie à l'ensemble des couples $(\delta, s)$ avec $\delta \in \Delta$ et $0 \leq s \leq k(\delta)$ avec l'équivalence

$$
[\delta, k(\delta)] \sim\left[g^{k(\delta)} \sigma, g^{h(\sigma)} w\right]=q\left[\theta \sigma, g^{k(\sigma)} w\right]=\theta \sigma .
$$

On voit donc que $D$ s'identifie au flot spécial $[\Delta, k]$ et que la transformation induite $\tilde{\theta}$ sur $\Delta$ commute avec $\Gamma$, en étant fibrée au-dessus de $\theta$. L'analyse du flot $g^{t}$ sur $W$ ou $D$ va alors se réduire à celle de la transformation fibrée $\tilde{\theta}$. En fait l'espace $\Sigma$ admet des partitions finies de diamètre arbitrairement petit formées d'ensembles ouverts et fermés. Le fibré holdérien $\Delta$ se réduit donc, par le choix d'une section holdérienne à un produit $\Sigma \times \Gamma$ et la transformation $\tilde{\theta}$ s'écrit: $\theta[\sigma, \gamma]=[\theta \sigma, \gamma+f(\sigma)]$ avec une certaine fonction holdérienne $f$, à valeurs dans $\Gamma$, définie à homologie près. D'autre part la mesure $\mu$, correspond par le codage $\eta$ à une mesure invariante sur $C$ laquelle induit une mesure $\theta$-invariante sur $\Sigma$ que l'on notera $\pi$; d'autre part $\mu$ s'exprime aisément à partir de $\pi$. Si alors $A_{n}=\sum_{0}^{n-1} h \circ \theta^{k}$ désigne le $n^{\text {ième }}$ temps de retour du flot $g^{t}$ dans $\Sigma$, la quantité $(1 / A) \gamma_{A}$ aura une limite quand $A$ tend vers l'infini si elle en possède une quand $A=A_{n}$ et $n$ tend vers l'infini. Or $\gamma_{A_{n}}(v)$ diffère de $\sum_{0}^{n-1} f \circ \theta^{k}(\sigma)$ par une quantité bornée lorsque $v=\eta\left[g^{s} \sigma\right][0 \leq s \leq h(\sigma)]$. On en conclut, d'après le théorème ergodique appliqué à $f, h$ et en tenant compte de l'ergodicité de $\mu, \pi$ :

$$
\lim _{n}\left(1 / A_{n}\right) \gamma_{A_{n}}=\left(\int f(\sigma) d \pi(\sigma)\right) /\left(\int h(\sigma) d \pi(\sigma)\right) \mu-p . p .
$$

Ceci montre l'existence du nombre de tours $e_{\mu}^{w}$ pour $g^{t}$ et $\mu$-presque tout $v$. 
On notera dans la suite $(P)$ la condition que les trajectoires périodiques du flot $\boldsymbol{g}^{t}$ sur $\Lambda$ représentent tous les éléments de $\Gamma$, c'est-à-dire:

Condition $P$ :

$\forall \gamma \in \Gamma, \exists v \in \Lambda$ et $\tau \in \mathbb{R}$

avec

$$
g^{\tau} w=\gamma w \quad \text { si } p(w)=v .
$$

(2) Ergodicité de flots en mesure infinie. On peut maintenant énoncer le

THÉORÈME. Soit $\left(V, g^{t}\right)$ un flot différentiable sur une variété riemannienne $V, \Lambda$ un compact $g^{t}$-invariant qui est un ensemble basique hyperbolique, $\mu$ une mesure de Gibbs sur $\Lambda$. Soit $W$ un revêtement abélien de $V$ à fibre $\Gamma \sim \mathbb{Z}^{d}$ et $\tilde{\mu}$ la mesure relevée de $\mu$ sur $W$; on suppose que les trajectoires périodiques de $\mathrm{g}^{t}$ représentent tous les éléments de $\Gamma$ (condition $P$ ). Alors les deux conditions suivantes sont équivalentes.

(1) Le flot $\left(W, g^{t}, \tilde{\mu}\right)$ est ergodique.

(2) $e_{\mu}^{w}=0$ et $d \leq 2$.

Preuve. Les considérations précédentes montent que l'ergodicité de $\left(W, g^{\prime}, \mu\right)$ équivaut à celle de $(\Delta, \tilde{\theta}, \tilde{\pi})$ où $\tilde{\pi}$ est la mesure relevée de $\pi$ dans $\Delta$. En cas d'ergodicité, on a donc, d'après le paragraphe précédent: $\int f d \pi=0, d \leq 2$ donc aussi $e_{\mu}^{w}=0$. Inversement, supposons ces conditions réalisées et $(\Delta, \tilde{\theta}, \tilde{\pi})$ non ergodique; ceci implique d'après le paragraphe précédent l'existence d'une fonction $\varphi$ holdérienne sur $\Sigma$ et d'un caractère $\chi$ de $\Gamma$ tels que $\varphi \cdot \chi$ soit $\tilde{\theta}$-invariante. On peut alors définir $\psi$ holdérienne sur $D$ avec $g^{t} \psi=\psi, \psi(\gamma \cdot d)=\chi(\gamma) \psi(d)=\forall t \in \mathbb{R}, \gamma \in \Gamma$, $d \in D$ : il suffit de poser $\psi(d)=\varphi(\delta)$ si $d=g^{t} \delta(t \in \mathbb{R}, \delta \in \Delta)$. Montrons que $\chi\left(\gamma^{N}\right)=1$ $\forall \gamma \in \Gamma$ où $N$ est le cardinal maximum de l'image réciproque par $\eta$ d'un point de $\Lambda$. Soit $v \in \Lambda$ tel que l'orbite de $v$ soit périodique de période $\tau$ et représente $\gamma \in \Gamma$, $c \in C$ avec $\eta(c)=v$. On a alors $\eta\left(g^{\tau} c\right)=v$, et $g^{\tau}$ est donc une permutation de l'ensemble fini $\eta^{-1}\{v\}$; on en déduit $g^{\tau N}(c)=c$ et si $d \in D$ se projette sur $c$, on aura $\gamma^{N} d=g^{\tau N} d$. Cette dernière relation donne $\chi\left(\gamma^{N}\right)=1$ puisque

$$
\psi(\gamma \cdot d)=\chi(\gamma) \psi(d)
$$

On peut maintenant remplacer $W$ et $D$ par des fibrés quotients $W_{1}$ et $D_{1}$ de fibres finies s'identifiant à $\Gamma_{1}=\Gamma / \Gamma^{N}$. Considérons dans $W_{1}$ les ensembles définis par $\psi=$ cte: ils forment une partition en fermés de $D_{1}$ et chacun d'eux se projette sur $C$ en un fermé $g^{t}$ invariant. Donc l'une au moins de ces projections fermées est de mesure pleine par ergodicité; comme les mesures de Gibbs chargent ici tous les ouverts, on en conclut que l'un des ensembles $\psi=$ cte se projette sur $C$ tout entier; cet ensemble définit une section de $D_{1}$ puisque $\psi(\gamma d)=\gamma(\gamma) \psi(d)$, section qui identifie $D_{1}$ au produit $C \times \Gamma_{1}$. On en déduit que $W_{1}$ s'identifie aussi à un produit $\Lambda \times \Gamma_{1}$, ce qui contredit la condition $P$.

(3) Flot géodésique. On considère ici l'espace hyperbolique $H$ de dimension $n$ dont le groupe des isométries positives s'identifie au groupe orthogonal $\operatorname{SO}(n, 1), \Gamma_{0}$ un sous-groupe discret opérant sans points fixes sur $H$. On identifiera $H$ à l'intérieur de la sphère unité $S^{n-1}$.

L'exemple classique des groupes de Schottsky est le suivant: on considère $2 p$ 'hémisphères solides' orthogonales à $S^{n-1}$ et disjointes $u_{1}, u_{2}, \ldots, u_{2 p}$ et on considère 
l'isométrie $a_{i}(1 \leq i \leq p)$ transformant l'extérieur de $u_{k}$ en l'intérieur de $u_{p+k}$. Le complémentaire de l'union des $u_{i}(1 \leq i \leq 2 p)$ est alors un domaine fondamental pour le groupe $\Gamma_{0}$ qui est engendré par les $a_{i}$, est libre et discret.

On rappelle que l'ensemble $L$ des points limites de $\Gamma_{0}$ est le fermé de $S^{n-1}$ formé des points d'accumulation d'une orbite $\Gamma_{0} a(a \in H)$. On considère la partie $A$ de $H$ qui est l'union des géodésiques de $H$ joignant 2 points distincts de $L$ et $\hat{A}$ l'ensemble des vecteurs unitaires correspondants. Il est clair que $\hat{A}$ est un fermé stable par $\Gamma$ et par le flot géodésique $g^{t}(t \in \mathbb{R})$. Considérons le quotient du fibré tangent unitaire $\hat{H}$ de $H$ par $\Gamma_{0}$ et en particulier les quotients $\Gamma_{0} \backslash A_{\Gamma_{0}} \backslash H$ et $\Gamma_{0} \backslash \hat{A} \subset \Gamma_{0} \backslash \hat{H}$. On vérifie aisément que l'ensemble des points non errants de $\Gamma_{0} \backslash \hat{H}$ sous l'action du flot géodésique est égale à $\Gamma_{0} \backslash \hat{A}$ et il est classique [7] que $g^{t}$ est topologiquement transitif sur $\Gamma_{0} \backslash \hat{A}$ comme $\Gamma_{0}$ est topologiquement transitif sur $L \times L$ privé de sa diagonale. On fera ici l'hypothèse que $\Gamma_{0}$ est convexe-cocompact et on en rappelle la dèfinition [24].

Définition. On dit que $\Gamma_{0}$ est convexe-cocompact s'il existe dans $A$ un compact $C$ tel que

$$
A=\bigcup_{\gamma \in \Gamma_{0}} \gamma C .
$$

Cette condition est par exemple réalisée pour les groupes de Schottsky et plus généralement par les groupes ayant un domaine fondamental à un numbre fini de faces qui ne rencontre pas $L$ [24].

Dans cette hypothèse l'ensemble $\Lambda={ }_{\Gamma_{0}} \backslash \hat{A}$ est évidemment un ensemble hyperbolique de $\Gamma_{0} \backslash \hat{H}$. Les géodésiques périodiques de $\Gamma_{0} \backslash H$ correspondent aux géodésiques laissées invariantes par un élément de $\Gamma_{0}$, et joignent donc deux points de $L$ laissés fixes par un élément de $\Gamma_{0}$. Par densité de ces points fixes dans $L$, ces géodésiques sont denses dans $\Gamma_{\Gamma_{0}} \backslash A$; il en est de même pour les trajectoires périodiques du flot $g^{\prime}$ sur $\Gamma_{0} \backslash \hat{A}$ et l'axiome $A$ de Smale est vérifié. En fait l'ensemble $\Gamma_{0} \backslash \hat{A}$ est basique hyperbolique d'après le théorème de décomposition spectrale de Smale [21] puisqu'il est connexe par transitivité topologique et ne possède pas de point fixe. C'est également un attracteur au sens de [4]. Si $\Gamma_{1}$ est un sous-groupe normal de $\Gamma_{0}$ la variété $\Gamma_{1} \backslash \hat{H}$ est un revêtement de $\Gamma_{0} \backslash \hat{H}$ et $\Gamma_{1} \backslash \hat{A}$ est fibré au-dessus du compact $\Gamma_{0} \backslash \hat{A}$ de fibres isomorphes à $\Gamma_{1} \backslash \Gamma_{0}$.

Donnons-nous une fonction holdérienne $F$ sur $_{\Gamma_{0}} \backslash H$ : elle définit un état d'équilibre sur $\Gamma_{0} \backslash \hat{A}$ qui est une mesure de probabilité maximisant la somme de l'entropie de $g^{x}$ et de l'intégrale $\int F d \mu$. Cette mesure se relève canoniquement dans $\Gamma_{1} \backslash \hat{A}$ en une mesure $\tilde{\mu}$ et le théorème suivant permet de rétrouver les résultats de [18].

THÉORÈME. Soit $\Gamma_{0}$ un sous-groupe discret d'isométries de l'espace hyperbolique $H$ qui est convexe-cocompact, $F$ une fonction holdérienne sur $\Gamma_{0} \backslash H$ et $\mu$ l'état d'équilibre correspondant sur $\Gamma_{0} \backslash \hat{A}$. Soit $\Gamma_{1}$ un sous-groupe distingué de $\Gamma_{0}$ telque $\Gamma_{0} / \Gamma_{1}=\mathbb{Z}^{d}$ et $\tilde{\mu}$ la mesure relevée de $\mu$ sur $\Gamma_{1} \backslash A$. Alors le flot géodésique $g^{t}$ sur $r_{1} \backslash \hat{A}$ est ergodique si et seulement si $d \leq 2$.

Preuve. Il suffit de vérifier les hypothèses du théorème du paragraphe précédent, la 
variété $V$ étant ici $\Gamma_{0} \backslash \hat{H}, \Lambda={ }_{\Gamma_{0}} \backslash \hat{A}, \Gamma=\Gamma_{0} / \Gamma_{1}$ et $W={ }_{\Gamma_{1}} \backslash \hat{H}$. La condition $(P)$ est évidemment vérifiée puisque toute géodésique périodique se relève dans $H$ en une géodésique dont les extrémités sont échangées par un élément de $\Gamma$. L'application de $\Gamma_{0} \backslash \hat{A}$ dans $\Gamma_{0} \backslash \hat{A}$ qui associe à un vecteur unitaire tangent $v$ le vecteur opposé- $v$ préserve la mesure $\mu$ d'après l'unicité de l'état d'équilibre correspondant à $F$ et la relation $F(v)=F(-v)$. La définition du nombre de tours $e_{\mu}^{w}$ à l'aide d'une trajectoire du flot montre donc que ici: $e_{\mu}^{w}=-e_{\mu}^{w}$ donc $e_{\mu}^{w}=0$. Dès lors la condition nécessaire et suffisante d'ergodicité du flot géodésique relativement à $\tilde{\mu}$ sur $W=\Gamma_{1} \backslash \hat{A}$ se réduit à $d \leq 2$.

\section{Remarques}

(1) La mesure d'entropie maximale pour le flot $g^{\prime}$ est l'état d'équilibre correspondant à la fonction nulle. Elle vérifie donc l'hypothèse de nullité du nombre de tours et on a l'ergodicité de $g^{\prime}$ sur $_{\Gamma_{1}} \backslash \hat{A}$ par rapport à cette mesure. En [24] est construite une mesure $\operatorname{sur}_{\Gamma_{0}} \backslash \hat{A}$ à partir d'une 'densité conforme' sur $L$ ou 'mesure de Patterson'. On peut voir d'après les propriétés d'équidistribution des géodésiques [3] $\operatorname{sur} \Gamma_{0} \backslash \hat{A}$ que cette mesure coïncide avec la mesure d'entropie maximale.

(2) Il est possible de montrer, grâce aux techniques de [10] que si $\Gamma_{0} / \Gamma_{1}$ est remplacé par un sous-groupe d'un groupe linéaire, le résultat final est inchangé: $\Gamma_{0} / \Gamma_{1}$ doit être essentiellement isomorphe à $\mathbb{Z}^{2}$ ou $\mathbb{Z}^{1}$. Le résultat de [25] montre que c'est probablement le seul cas possible d'ergodicité, sans hypothèse particulière sur la structure de $\Gamma_{0} / \Gamma_{1}$.

\section{Appendice}

On établit ici, brièvement, le théorème limite local de [8], qui a été utilisé dans la preuve du Corollaire 3 au second théorème du paragraphe 2.4. On discute aussi de la validité d'estimations voisine en relation avec le propriétés $K$ et 'non-Bernouilli'.

On conserve les notations déjà introduites: $(\Sigma, \theta, \pi)$ est un sous-shift de type fini, de partie positive $\left(\Sigma^{+}, \theta, \pi^{+}\right), f$ est une fonction Holdérienne à valeur dans $\mathbb{R}^{d}$ que l'on peut supposer définie sur $\Sigma^{+}, P$ est l'adjoint de $\theta, \tilde{P}$ celui de $\tilde{\theta}$ et $P_{\lambda}$ est défini $\operatorname{par} P_{\lambda} \varphi(x)=P\left[e^{i\langle\lambda, f\rangle} \varphi\right]$ où $\lambda \in \mathbb{R}^{d}$ et $\varphi$ est définie sur $\Sigma^{+}$.

ThÉORÈme. Avec les notations précédentes, supposons $f$ strictement apériodique et d'intégrale nulle. Alors, pour tous $(x, t) \in \Sigma^{+} \times \mathbb{P}^{d}$, la suite de mesures $n^{d / 2} \tilde{P}^{n}[(x, t), \cdot]$ converge vaguement vers une multiple de la mesure $\pi^{+} \times 1$.

Preuve. On peut tester la convergence vague sur les fonctions de la forme $\varphi \cdot \psi$ ou $\varphi$ est holdérienne sur $\mathbb{R}^{d}$ et telle que sa transformée de Fourier soit à support compact. Alors: $\tilde{P}^{n}[(x, t), \varphi \cdot \psi]=E_{x}\left[\varphi\left(x_{n}\right) \psi\left(t+S_{n}\right)\right]$ où $\left(x_{n}, S_{n}\right)$ est la position à l'instant $n$ de la trajectoire de la chaine de Markov de noyau $\tilde{P}$. De plus:

$$
\psi\left(t+S_{n}\right)=\int e^{i\left\langle\lambda, t+S_{n}\right\rangle} \hat{\psi}(\lambda) d \lambda
$$

et: $E_{x}\left[\varphi\left(x_{n}\right) \psi\left(t+S_{n}\right)\right]=\int e^{i\langle\lambda, t\rangle} P_{\lambda}^{n} \varphi(x) \hat{\psi}(\lambda) d \lambda$. Comme $P_{\lambda}$ est quasi-compact [8], il admet une décomposition spectrale:

$$
P_{\lambda}=k(\lambda) \pi_{\lambda}+r_{\lambda}
$$


où $\pi_{\lambda}$ est un projecteur qui commute avec $r_{\lambda}$, lequel est de norme spectrale inférieure à 1 [cf 8].

$k(\lambda)$ étant de module strictement inférieur à 1 pour $\lambda \neq 0$. On obtient alors:

$$
n^{d / 2} E_{x}\left[\varphi\left(x_{n}\right) \psi\left(t+S_{n}\right)\right]=\int e^{i(\lambda / \sqrt{ }, t)} \hat{\psi}(\lambda / \sqrt{n}) P_{\lambda / \sqrt{ } n}^{n} \varphi(x) d \lambda
$$

et la décomposition précédente donne:

$$
P_{\lambda / \sqrt{n}}^{n} \varphi(x) \sim k^{n}(\lambda / \sqrt{n}) \sim e^{-\sigma^{2} \lambda^{2} / 2},
$$

où $\sigma^{2}$ est un nombre strictement positif. On a donc la convergence de $n^{d / 2} \tilde{P}^{n}[(x, t)$, $\varphi \cdot \psi]$ vers $\pi^{+}(\varphi) \hat{\psi}(0) \int e^{-\left(\sigma^{2} / 2\right) \lambda^{2}} d \lambda$, c'ést-à-dire $\pi^{+}(\varphi) l(\psi)\left(2 \pi \sigma^{2}\right)^{d / 2}$.

On a alors le corollaire suivant qui donne la première estimation (2.1) du travail de S. Kalikow [12].

Corollaire. Soit $f$ une fonction holdérienne de $\Sigma$ dans $\mathbb{R}$ qui est strictement apériodique, d'intégrale nulle et posons:

$$
S_{n}(\omega)=\sum_{0}^{n-1} f_{0} \theta^{k}(\omega)
$$

alors il existe une constante $c>0$ telle que pour tous $n \in \mathbb{N}$ et $I \in \mathbb{R}$ :

$$
\pi\left\{\left|S_{n}(\omega)\right| \leq I\right\} \leq C \frac{I}{\sqrt{n}}
$$

Preuve. On se ramène comme dans [8] à une fonction $f$ définie sur $\Sigma^{+}$. On peut majorer l'indicateur de $\left[-\frac{1}{2}, \frac{1}{2}\right]$ par une fonction $\psi$ dont la transformée de Fourier est à support compact et on observe alors que pour tout $t \in \mathbb{R}$ :

$$
\begin{gathered}
\pi\left\{S_{n}(\omega) \in\left[t-\frac{1}{2}, t+\frac{1}{2}\right]\right\} \leq \int \tilde{P}^{n}[(x, t), 1 \cdot \psi] d \pi^{+}(x), \\
\pi\left\{S_{n}(\omega) \in\left[t-\frac{1}{2}, t+\frac{1}{2}\right]\right\} \leq \int|\hat{\psi}(\lambda)|\left|P_{\lambda}^{n} 1(x)\right| d \lambda d \pi^{+}(x)
\end{gathered}
$$

et ceci donne d'après la preuve précédente l'estimation indépendente de $t$ :

$$
\pi\left\{S_{n}(\omega) \in\left[t-\frac{1}{2}, t+\frac{1}{2}\right]\right\} \leq C / 4 \sqrt{n} .
$$

Enfin, recouvrant l'intervalle $[-I, I]$ par $2 l+1$ intervalles de la forme $\left[k-\frac{1}{2}, k+\frac{1}{2}\right]$ $(k \in \mathbb{Z})$ on obtient:

$$
\pi\left\{\left|S_{n}(\omega)\right| \leq I\right\} \leq C I / \sqrt{n} .
$$

La relation: $\lim _{n} 1 / n \int\left|S_{n}(\omega)\right|^{2} d \pi(\omega)=\sigma^{2}>0$ est classique dans la preuve du théorème central limite laquelle est en fait contenue dans celle du théorème local [cf 8]. On en déduit aisément l'estimation (2.2) de [12] d'après l'inégalité de Tchebitcheff:

$$
\pi\left\{\left|S_{n}(\omega)\right|>I\right\} \leq 2 C^{\prime} n / I^{2} \text { avec } C^{\prime}>\sigma^{2} .
$$

L'inégalité (2.3) de [12]:

$$
\pi\left\{\sup _{I \leq k \leq n}\left|S_{k}(\omega)\right|>I\right\} \leq C^{\prime \prime} n / I^{2}
$$


peut être également obtenue ici en suivant la méthode de [14] et ses estimations qui permettent d'obtenir le principe d'invariance et des théorèmes limites locaux renforcés pour des produits de matrices aléatoires.

On obtient donc ainsi, grâce à ces trois inégalités, les estimations basiques 2.5 et 2.6 de [12].

On peut enfin, suivant les méthodes de [8], établir une loi des grands écarts du type (2.4) qui est généralement nécessaire pour suivre la preuve de [12], mais son utilisation paraît moins aisée.

Etablissons, en particulier, l'inégalité (2.3) de [12]:

$$
\pi\left\{\sup _{I \leq k \leq n}\left|S_{k}(\omega)\right|>I\right\} \leq C^{\prime \prime} n / I^{2} .
$$

Modifiant quelque peu les notations, on considère un espace compact $X$, un noyau Markovien $P(x, d y)$ sur $X$, de trajectoires $=\left(x_{k}\right)_{k \in N}$, une fonction continue $f$ sur $X$ et les sommes de Birkhoff:

$$
S_{n}(\Xi)=\sum_{1}^{n} f\left(x_{k}\right)=\sum_{1}^{n} X_{k}
$$

On suppose fixée une mesure invariante $\pi$ pour le noyau $P, \int f d \pi=0$ et l'on note:

$$
S_{n}^{*}(\Xi)=\sup _{1 \leq k \leq n}\left|S_{k}(\Xi)\right|
$$

On a alors le:

THÉORÈME. Supposons que la fonction $f$ satisfasse uniformément le théorème central limite avec reste: il existe une constant $C$ telle que pour tous $x \in X, t \in \mathbb{R}, n \in N$ on ait:

$$
\left|P_{x}\left\{S_{n}<\sigma t \sqrt{n}\right\}-\frac{1}{\sqrt{2 \pi}} \int_{-\infty}^{1} e^{-u^{2} / 2} d u\right| \leq \frac{C}{\sqrt{n}} .
$$

Alors, il existe un réel $d \geq 0$ tel que pour tous $x \in X, b>0, n \in N$ on ait:

$$
\mid P_{x}\left\{S_{n}^{*} \geq b\right\} \leq 4 P_{x}\left\{\left|S_{n}\right| \geq b-d \sqrt{n}\right\}
$$

La preuve s'appuie sur le lemme.

Lemme. Soient y et a deux réels donnés. Alors on a pour tous $x$ de $X, n$ entier:

$$
P_{x}\left\{\left|S_{n}\right|>y\right\} \geq P_{x}\left\{S_{n}^{*} \geq y+a\right\} \underset{z \in X, k \in N}{\operatorname{Inf}} P_{z}\left\{\left|S_{k}\right|<a\right\}
$$

Afin de prouver le lemme, introduisons les ensembles:

$$
\begin{gathered}
C_{n}=\left\{\left|S_{n}\right|>y\right\}, \quad B_{k}=\left\{\left|\sum_{k+1}^{n} X_{p}\right|<a\right\}, \\
A_{k}=\left\{\left|S_{j}\right|<y+a \forall_{j}<k,\left|S_{k}\right| \geq y+a\right\} .
\end{gathered}
$$

On a clairement:

$$
C_{n} \supset \bigcup_{k \leq n}\left(A_{k} \cap B_{k}\right) \text { et } P_{x}\left(C_{n}\right) \geq \sum_{k \leq n} P_{x}\left(A_{k} \cap B_{k}\right)
$$




$$
\begin{aligned}
& P_{x}\left(A_{k} \cap B_{k}\right)=\int 1_{A_{k}}(\Xi) 1_{B_{k}}(\Xi) d P_{x}(\Xi), \\
& P_{x}\left(A_{k} \cap B_{k}\right)=\int 1_{A_{k}}(\Xi) P_{x_{k}}\left(B_{k}\right) d P_{x}(\Xi), \\
& P_{x}\left(A_{k} \cap B_{k}\right) \geq \operatorname{Inf}_{x \in X} P_{x}\left\{\left|S_{n-k}(\Xi)\right|<a\right\} P_{x}\left(A_{k}\right),
\end{aligned}
$$

d'où la relation voulue puisque:

$$
P_{x}\left\{S_{n}^{*} \geq y+a\right\}=\sum_{k \geq n} P_{x}\left(A_{k}\right) .
$$

Preuve du théorème. On prend $a=d \sqrt{n}$ dans le lemme et on va trouver $d$ :

$$
P_{z}\left\{\left|S_{k}\right|<d \sqrt{n}\right\} \geq P_{z}\left\{\frac{\left|S_{k}\right|}{\sqrt{k}}<d\right\} \geq \frac{1}{\sigma \sqrt{2 \pi}} \int_{-d}^{d} e^{-u^{2} / 2 \sigma^{2}} d u-\frac{C}{\sqrt{k}}
$$

d'après l'hypothèse du théorème. Pour $k>n_{0}$, le terme $C / \sqrt{k}$ est majoré par $\frac{1}{4}$. Fixons $d$ de façon que:

$$
\frac{1}{\sigma \sqrt{2 u}} \int_{-d}^{d} e^{-u^{2} / 2 \sigma^{2}} d u>\frac{1}{2} \quad \text { et } d>\sqrt{n_{0}}\|f\|_{\infty} .
$$

La dernière condition assure que:

$$
\forall z \in X \quad P_{z}\left\{\left|S_{k}\right|<d \sqrt{n}\right\}=1 \quad \text { pour } k \leq n_{0} .
$$

On a donc finalement, pour tout $k$ et tout $z$ de $X$ :

$$
P_{z}\left\{\left|S_{k}\right|<d \sqrt{n}\right\} \geq \frac{1}{4} \text {. }
$$

Le lemme donne alors, avec $a=d \sqrt{n}$

$$
P_{x}\left\{S_{n}^{*}>b\right\} \leq 4 P_{x}\left\{\left|S_{n}\right|>b-d \sqrt{n}\right\}
$$

Corollaire 1. Supposons que, avec les hypothèses du théorème, il existe $\mathrm{c}>0$ avec $E_{x}\left(\left|S_{n}\right|^{2}\right) \leq c n$. Alors il existe $C>0$ tel que pour tous $x \in X, b \in \mathbb{R}^{+}, n \in N$ :

$$
P_{x}\left\{S_{n}^{*}>b\right\} \leq C \frac{n}{b^{2}} .
$$

Preuve. Il suffit de justifier l'inégalité pour $n / b^{2}$ assez petit; le cas général s'obtient en modifiant la constante $C$.

On prend ici $n / b^{2}<1 / 4 d^{2}$ où $d$ est défini au théorème précédent. Ce théorème donne:

$$
P_{x}\left\{S_{n}^{*} \geq b\right\} \leq 4 P_{x}\left\{\left|S_{n}\right| \geq b-d \sqrt{n}\right\} \leq 4 \frac{n c}{(b-d \sqrt{n})^{2}}
$$

en appliquant l'inégalité de Tchebitcheff.

Or ici:

$$
\frac{n}{(b-d \sqrt{n})^{2}} \leq \frac{n}{\left(b-\frac{b}{2}\right)^{2}}=\frac{4 n}{b^{2}}
$$

Au total:

$$
P_{x}\left\{S_{n}^{*} \geq b\right\} \leq 16 c \frac{n}{b^{2}}
$$


COROllaire 2. Soit $(\Sigma, \theta, \pi)$ un sous-shift de type fini, $f$ une fonction holdérienne réelle, strictement apériodique et d'intégrale nulle et posons:

$$
S_{n}(\omega)=\sum_{1}^{n} f \circ \theta^{k}(\omega) .
$$

Alors il existe une constante $C$ telle que tous $u \in N$ et $I \in \mathbb{R}^{+}$, on ait:

$$
\pi\left\{S_{n}^{*}(\omega) \geq I\right\} \leq \mathrm{Cn} / I^{2} .
$$

Preuve. Il suffit encore de prouver l'estimation pour $n / I^{2}$ assez petit.

La fonction $f$ s'écrit avec $u$ Holdérienne:

$$
f=g+u \circ \theta-u \text {. }
$$

Notant $T_{n}(\omega)=\sum_{1}^{n} g \circ \theta^{k}(\omega)$, la loi de $T_{n}$ est la même que celle d'une somme de Birkhoff pour le noyau adjoint $P$ sur $X=\Sigma^{+}$et la fonction $g$.

Les hypothèses du Corollaire 1 étant satisfaites pour la fonction $g$ en raison d'estimations analogues à celles du Théorème 1, développées en [8] et fournissant le théorème central limite avec reste, on a:

$$
\pi\left\{T_{n}^{*}(\omega) \geq b\right\} \leq C_{1} \frac{n}{b^{2}} \text { pour une certaine constante } C .
$$

Ici, on a clairement $\left|S_{n}^{*}-T_{n}^{*}\right| \leq 2\|u\|_{\infty}=\alpha$. On prendra ici $n / I^{2} \leq 1 / 4 \alpha^{2}$, de sorte que, en particulier $I \geq 2 \alpha$ et:

$$
\pi\left\{S_{n}^{*} \geq I\right\} \leq\left\{T_{n}^{*} \geq I-\alpha\right\} \leq C_{1} \frac{n}{(I-\alpha)^{2}} \leq 4 C_{1} \frac{n}{I^{2}} .
$$

\section{REFERENCES}

[1] R. L. Adler \& P. C. Shields. Skew products of Bernoulli shifts with rotations. Israël J. Math. 19 (1974), 228-236.

[2] R. Bowen. Equilibrium states and the ergodic theory of Anosov diffeomorphisms. Lecture Notes in Mathematics 470 (Springer-Verlag, Berlin, 1975).

[3] R. Bowen. Symbolic dynamics for hyperbolic flows. Amer. J. Math. 95 (1973), 429-460.

[4] R. Bowen \& D. Ruelle. The ergodic theory of axiom A flows. Inventiones Math. 29 (1975), 181-202.

[5] L. A. Bunimovich \& Ya G. Sinai. Statistical properties of Lorentz gas with periodic configurations. Commun. Math. Phys. 78 (1981), 479-497.

[6] G. Choquet \& J. Deny. Sur l'équation de convolution $\mu=\sigma * \mu "$. C.R.A.S. Paris 250A (1960), 799-801.

[7] W. H. Gottschalk \& G. A. Hedlund. Topological dynamics. Amer. Math. Soc. 36 (1955).

[8] Y. Guivarc'h \& J. Hardy. Théorèmes limites pour une classe de chaînes de Markov et applications aux difféomorphismes d'Anosov. Annales de l'Inst. H. Poincaré 24 (1) (1988), 73-98.

[9] Y. Guivarc'h. Applications d'un théorème limite local à transience et à la récurrence de marches de Markov. Théorie du Potentiel. Lecture Notes in Mathematics itgıcb1096 (Springer-Verlag, Berlin, 1983). 301-332.

[10] Y. Guivarc'h, M. Keane \& B. Roynette. Marches aléatoires sur les groupes de Lie. Lecture Notes in Mathematics 624 (Springer-Verlag, Berlin, 1977).

[11] Y. Guivarc'h. Mouvement brownien sur les revêtements d'une variété compacte. C.R.A.S. Paris 292 (1981) 851-853.

[12] S. Kalikow. $T, T^{-1}$ transformation is not loosely Bernoulli. Ann. Math. 115 (1982), 393-409.

[13] A. Katok. Smooth non-Bernoulli $K$-automorphisms. Inventiones Math. 61 (1980), 291-300. 
[14] E. Le Page. Théorèmes limites pour les produits de matrices aléatoires. Lecture Notes in Mathematics 928, (Springer-Verlag, Berlin, 1982) 355-386.

[15] A. N. Lifshits. Homology properties of Y-systems. Math. Zametki 10 (5) (1971), 555-564.

[16] M. Lin. Mixing for Markov operators. Z. Wahr. 19 (1971), 231-242.

[17] I. Meilijson. Mixing properties of a class of skew-products. I. J. Math. 19 (1974), 266-270.

[18] M. Rees. Checking ergodicity of some geodesic flows with infinite Gibbs measure. Ergod. Th. \& Dynam. Sys. 1 (1981), 107-133.

[19] D. J. Rudolph. $\mathbb{Z}^{n}$ and $\mathbb{R}^{n}$ cocycle extensions and complementary algebras. Preprint Depart. of Math., University Maryland.

[20] D. J. Rudolph. Asymptotically brownian skew products give non loosely Bernoulli $K$-automorphisms. Preprint. University of Maryland.

[21] S. Smale. Differentiable dynamical systems. Bull. Amer. Math. Soc. 73 (1967), 747-817.

[22] Ya G. Sinai. Gibbs measures in ergodic theory. Russian Math. Surveys 27 (4) (1972), 21-69.

[23] F. Spitzer. Principles of random walks. Van Nostrand, Princeton, 1964.

[24] D. Sullivan. The density at infinity of a discrete groupe of hyperbolic motions. I.H.E.S. Publ. Math. 50 (1979), 171-202.

[25] J.-P. Thouvenot. Communication personnelle.

[26] N. Th. Varopoulos. C.R.A.S. Paris 302 (1) 6 (1986), 203-205. 